\title{
Nuovo periodo
}

Giovanni Lodi

Dipartimento di Scienze Biomediche, Chirurgiche e Odontoiatriche

Università degli Studi di Milano

via Beldiletto 1/3 Milano 20142

Italia

giovanni.lodi@unimi.it

tel. + 390250319021

fax. +390250319041

Poche settimane fa ad Amsterdam si sono incontrati oltre 10 mila tra odontoiatri e igienisti, giunti in Olanda da oltre cento nazioni. L'occasione era Europerio, l'appuntamento più importante per i parodontologi europei e non solo. Chi c'è stato, mi ha raccontato di un congresso grande e bello, in cui come al solito clinici e ricercatori italiani sono stati tra i protagonisti.

Uno dei motivi di maggiore interesse del congresso è stata la presentazione della nuova classificazione delle malattie parodontali, enunciata in quattro consensus report, elaborati da un gruppo di esperti sulla base di una ventina di articoli che sintetizzano le conoscenze attuali sull'intera materia. Decine di pagine per descrivere la grande complessità di questo gruppo di patologie.

Cambia profondamente la parodontite: non più gruppo di condizioni distinte per aggressività o età di insorgenza, ma una malattia unica, con stadi e gradi diversi, determinati da gravità della stessa e caratteristiche del paziente. Un sistema che come per altre stadiazioni ha il compito primario di guidare le scelte terapeutiche.

Novità letteralmente epocale è l'inclusione delle malattie dei tessuti perimplantari, problema sconosciuto fino a pochi lustri fa, oggi di riscontro quotidiano e destinato a crescere. E poi, finalmente, una definizione di gengivite che discrimina tra malattia e infiammazione gengivale.

La nuova classificazione vede anche la fine del concetto (e del termine) di ampiezza biologica, e l'introduzione di quello di forze occlusali traumatiche. Lo spazio di un editoriale non può bastare a raccontarvi quanto profonde sono le innovazioni introdotte, ma Dental Cadmos tornerà a occuparsene in un futuro prossimo.

Vale la pena però usare queste ultime battute per sottolineare che questo non è il risultato di un esercizio tassonomico, di mera catalogazione, perché l'impressione è che i colleghi che ci hanno lavorato avessero un obiettivo molto più complesso e ambizioso, quello di offrire un nuovo modo con cui guardare queste malattie, basato sulle conoscenze acquisite nei venti anni passati dall'ultima classificazione e sulle modifiche che nello stesso periodo hanno investito la realtà odontoiatrica.

Buona lettura 